Toscano y García, Guillermo. “Ondas que Ilevan idioma nacional. Las Lecciones de Florencio Garrigós en la Escuela del Aire”. Anclajes, vol. XXV, n. ${ }^{\circ} 3$, septiembrediciembre 2021, pp. II7-I35.

https://doi.org/I0.19137/anclajes-2021-25314

\title{
ONDAS QUE LLEVAN IDIOMA NACIONAL. LAS LECCIONES DE FLORENCIO GARRIGÓS EN LA ESCUELA DEL AIRE
}

\section{Guillermo Toscano y García}

Instituto de Lingüística

Universidad de Buenos Aires

Argentina

gtoscano@filo.uba.ar

ORCID: 0000-000I-6437-2768

Fecha de recepción: I5/06/202I | Fecha de aceptación: 29/07/2021.

Resumen: En 1940, el periodista, gramático y docente argentino Florencio Garrigós publica sus Lecciones de idioma nacional. El presente trabajo tiene el objetivo de analizar, desde la perspectiva de la historiografía lingüística, los rasgos específicos que esta obra presenta en la historia de la gramática escolar argentina. Para ello, reconstruye en primer lugar la trayectoria profesional de su autor; luego, presenta en sus rasgos generales la Escuela del Aire, primera experiencia de educación a distancia que utilizó el formato radiofónico en la Argentina. Finalmente, analiza las Lecciones, y demuestra que se trata de un texto innovador en al menos dos sentidos: por la innovación didáctica que propone y por la definida perspectiva que adopta frente a los fenómenos de variación lingüística propios de la Argentina, que valora positivamente.

Palabras clave: Florencio Garrigós; Escuela del Aire; gramática escolar argentina; siglo $X X$; historiografía lingüística.

\section{Waves that carry national language. The Lecciones of Florencio Garrigós at the Escuela del Aire}

\begin{abstract}
In 1940, the Argentine journalist, grammarian and teacher Florencio Garrigós published his Lecciones de idioma nacional. Within the framework of linguistic historiography, we aim to analyze the specific features that this work presents in the history of Argentine school grammar.To do this, we first reconstruct the professional career of its author; then, we present the Escuela del Aire, the first distance education experience that used the radio in Argentina. Finally, we analyze the text of the Lecciones, and argue that it is an innovative work in at least two senses: because of the didactic innovation that it proposes and because of the defined perspective it adopts regarding the Argentinian linguistic variation phenomena, which it values positively.
\end{abstract}

Keywords: Florencio Garrigós; Escuela del Aire;Argentinian school grammar; twentieth century; linguistic historiography. 


\title{
Ondas que transmitem a lingua nacional. As Lecciones de Florencio Garrigós na Escuela del Aire
}

\begin{abstract}
Resumo: Em 1940, o jornalista, gramático e professor argentino Florencio Garrigós publicou suas Lecciones de Lengua Nacional. No marco da historiografia linguística, nos propomos a analisar as particularidades que este trabalho apresenta na história da gramática escolar argentina. Visando tal objetivo, primeiro reconstruímos a carreira profissional do autor; a seguir, apresentamos as principais características da Escuela del Aire, a primeira experiência de educação a distância que utilizou o formato de rádio na Argentina. Por fim, analisamos as Lecciones e mostramos que se trata de um texto inovador em dois sentidos: pela inovação didática que propõe e pela perspectiva que adota sobre os fenômenos de variação linguística típicos da Argentina, que ele valora positivamente.
\end{abstract}

Palavras chave: Florencio Garrigós; Escuela del Aire; gramática escolar argentina; Século XX; historiografia da lingüística.

as Lecciones de idioma nacional, de Florencio Garrigós (circa
1880-1956), ocupan un lugar singular en la historia de la en-
señanza gramatical escolar en la Argentina. Publicadas en 1940, consisten en la versión escrita de una experiencia novedosa: el curso que Garrigós, en el marco de la Escuela del Aire, había ofrecido a comienzos de ese mismo año en la Radio del Estado. Pero la singularidad de las Lecciones no radica únicamente en el formato que adoptan para dar respuesta a las exigencias impuestas por esas nuevas tecnologías de la palabra; se debe también al criterio radical que establecen para valorar los rasgos propios de la lengua hablada en el país. Así, frente a una tradición escolar que a menudo había buscado ignorarlas o corregirlas, el texto de Garrigós constituye una reivindicación de las características que, como el voseo, el seseo o el yeísmo, informaban el habla de los argentinos.

En lo que sigue, reconstruimos algunas de las coordenadas más destacadas de la trayectoria educativa y periodística de Garrigós; presentamos luego el proyecto de la Escuela del Aire, la primera experiencia de educación a distancia que utilizaba el formato radiofónico; $y$, finalmente, analizamos los aspectos más relevantes de las Lecciones.

\section{Florencio Garrigós}

En la sesión que la Academia Argentina de Letras realiza el 25 de abril de 1957, el académico Ricardo Sánz-Hayes se propone destinar "unas palabras en homenaje a dos escritores recientemente fallecidos: los doctores Ángel Acuña y Florencio Garrigós" (171). Como se apresura a recordar, ninguno de ellos había formado parte de la Academia, pese a lo cual, entiende, es justo honrar su trayectoria. Así, luego de repasar los méritos literarios, históricos, filosóficos y pedagógicos de Acuña, se afana en declarar la diferente estirpe de Garrigós: "Abogado y periodista, el rudo, silencioso y pertinaz oficio cotidiano [...] le hizo 
actuar, no diré en una zona de sombra, pero sí poco propicia para difundir prestigio cuando el hábito del anónimo se convierte en segunda naturaleza" (171). La observación de Sáenz-Hayes traza de este modo una aparente contradicción entre firma y prestigio, por un lado, como rasgos que distinguirían a la élite letrada; y el carácter despersonalizado y poco lucido que distinguiría a la escritura en el periódico.

Al esbozar una biografía, Sáenz-Hayes confirma esa presentación inicial. Dueńo, dice, de una vocación "acendrada y recóndita" por la gramática y la filología, Garrigós se incorpora a la redacción de La Prensa "con una veintena de años" para suceder "la memoria y erudición de un señor chapeado a la antigua que imponía con su prestancia tanto como con su predicamento", "el doctor Matías Calandrelli" (172). Este había tenido a su cargo la sección "Informaciones gramaticales y filológicas" y había publicado, en 1917 y 1919, dos volúmenes que compilaban gran parte de esas columnas; en 1944, casi tres décadas más tarde, Garrigós, que se había hecho cargo de la sección bajo el nuevo nombre de "Gramaticales y filológicas", continuará esa tradición y presentará una recopilación de sus artículos en un volumen, Gramaticales y filológicas de 'La Prensa'. Los libros de Calandrelli y Garrigós evidencian la aspiración a una forma de trascendencia propia del libro impreso; pero, a la vez, buscan asociar la firma del autor a un cierto tipo de circulación del saber, que si no es el de la Academia y tampoco el de un ya consolidado campo científico, parece sin embargo reclamar para sí un cierto tipo de autoridad.

La actividad periodística de Garrigós se extiende por varias décadas y a través de diferentes medios: durante el veinte, escribe frecuentemente sobre cuestiones lingüísticas en El Hogar, y es responsable de la sección "El idioma castellano en la Argentina" que se publica en Caras y Caretas. En esas columnas, y en la tradición de Calandrelli, Garrigós destina breves textos a dar cuenta del correcto sentido o uso de una expresión.

Pero su campo de intervención no se limita a la prensa, sino que se extiende a la educación, y es también por su labor en este ámbito que obtiene reconocimiento. Así, por ejemplo, lo presenta el diario Crítica, cuando lo invita a responder una encuesta, que publica durante junio de 1927, sobre la posible existencia de un "idioma propio" de los argentinos: "Florencio Garrigós (hijo) es profesor de castellano en la Biblioteca del Consejo Nacional de Mujeres y del Colegio Nacional Juan Martín de Pueyrredón, autor de varios trabajos lingüísticos y de 'El idioma castellano en la Argentina', que viene publicando la revista Caras y Caretas" (Garrigós Encuesta 12).

Un texto sin firma publicado también en El Hogar poco más tarde, en 1928, permite reconstruir parcialmente la actividad de Garrigós en uno de los espacios en los que se desempeña como docente, la Biblioteca del Consejo Nacional de Mujeres, y al mismo tiempo sus ideas acerca de la enseñanza de la gramática. El artículo comienza recogiendo un tópico del discurso gramatical del período, que denuncia el envejecimiento de los métodos utilizados para la enseñanza de 
la gramática y proclama la necesidad de su renovación. A continuación, el texto observa que la reforma necesaria, que no ha ocurrido todavía en el sistema educativo argentino, encuentra ejemplo y provecho en las clases dictadas por Garrigós en la Biblioteca del Consejo:

No se ha dado todavía, para la enseñanza de la gramática, con los sistemas prácticos, sintéticos, preconizados por la pedagogía moderna. Por eso nos es grato [...] referirnos al sistema adoptado para el caso, en la clase de Gramática que funciona en el curso de estudios de la Biblioteca del Consejo Nacional de Mujeres, bajo la dirección del profesor señor Florencio Garrigós (hijo). (Escollos 21)

Según se indica, en 1927 la Biblioteca había dispuesto crear un "curso especial de redacción y ortografía como complemento del de dactilografía" que allí se venía ofreciendo, y había confiado su dictado a Garrigós, de quien el artículo destaca la capacidad de innovación pedagógica. Ese método de enseñanza busca conducir la atención de las alumnas no hacia un saber teórico sino hacia uno práctico, específicamente hacia el uso escrito de la lengua, con el propósito de deducir a partir de él los principios de la gramática:

De acuerdo con la opinión de aquellos autorizados pedagogos que sostienen que el conocimiento debe ser producto espontáneo de la actividad del educando y adquirirse por propia experiencia, el señor Garrigós, en su método de enseñanza, resolvió prescindir en absoluto de las definiciones y de la exposición teórica de las intrincadas reglas cuyas excepciones son, a veces, tantas, que hacen pesado su aprendizaje, y estableció, en consecuencia, que sus alumnas estudiaran los hechos gramaticales, prácticamente, en la realidad de la vida, en la lectura de diarios y revistas, deduciendo, de la observación constante y metódica de los mismos, los principios ortográficos y sintácticos. (Escollos 21)

De acuerdo con su propuesta, la formación lingüística de las alumnas debía proceder de la exposición reiterada a los estímulos lingüísticos ofrecidos por la prensa periódica: cada una debía formar "una carpeta de recortes" que coleccionara "gran copia de ejemplos ortográficos, de concordancia, etc., mediante lo cual llega a familiarizarse pronto con las reglas que rigen la construcción de las cláusulas en lo escrito"; un método que el autor anónimo se propone ilustrar mediante la presentación del trabajo realizado por "una de las alumnas más aventajadas de dicha clase" (incluyendo una fotografía de su cuaderno), básicamente ejercicios gramaticales realizados a partir de "ejemplos tomados de las páginas de la revista El Hogar" (21). El artículo concluye pronunciándose abiertamente a favor de la renovación pedagógica propuesta por Garrigós, un rasgo que impacta en su participación, una década después, en el proyecto estatal de la Escuela del Aire.

Con excepción de los trabajos que, durante las décadas siguientes, publica en diarios y revistas, registramos otro material destinado a la enseńanza gramatical, particularmente de la ortografía: el Encuadernador ortográfico que publica posi- 
blemente en 1930 junto con Francisco Camón Gálvez¹. En este texto, se propone nuevamente una perspectiva renovadora que se manifiesta contra la tradición memorística y a favor de un saber práctico: "No se insista en el enunciado de la regla ortográfica, sino después que multitud de ejemplos hayan demostrado su conveniencia de manera inconcusa. Más que la exposición teórica, de dudoso resultado, la ortografía exige, imperiosamente, una práctica constante e ininterrumpida (Garrigós y Camón Gálvez: s/p).

El Encuadernador parece obtener una recepción favorable en el ámbito educativo, a juzgar por la reseña que le dedica El Monitor de la Educación Común, que, tras declarar que "la enseñanza de la gramática en los colegios nacionales, sigue siendo, para muchos profesores, un problema sin solución racional" recomienda abiertamente su uso: "El método que Garrigós y Camón Gálvez preconizan $-y$ que es justo y necesario recomendar a profesores y alumnos- se basa [...] en la doble acción que obliga a investigar a estos y ayuda a resolver a aquellos" (295).

De este modo, cuando, casi una década más tarde, Garrigós sea designado para dictar las lecciones de idioma nacional en el marco de la Escuela del Aire, lo hará ya investido de un prestigio que proviene no solamente de su extensa trayectoria como docente y en la prensa periódica, sino de un rasgo que señaladamente se le atribuye desde distintos ámbitos públicos, incluyendo los del Estado: su capacidad de innovar en materia de enseñanza de la lengua.

\section{La Escuela del Aire}

La Escuela del Aire se crea por un decreto presidencial publicado en el Boletín oficial el 31 de agosto de 1939, durante la presidencia de Roberto M. Ortiz y la gestión de Jorge Eduardo Coll como Ministro de Justicia e Instrucción Pública. Se trata, como ha sido ya observado por Nieto y de Majo, de una iniciativa pionera en la Argentina en el campo de la educación a distancia mediante el uso de la radiofonía. La disposición presidencial establecía la creación de una "comisión" que tendría a su cargo la preparación de un plan de funcionamiento para una futura "Escuela del Aire"; entre sus considerandos, señala que el uso de las transmisiones radiofónicas podría "constituir un medio eficaz de desarrollo cultural y un complemento de la función docente del maestro y profesor”. Según la misma disposición, la comisión sería tripartita (el Director General de Correos y Telégrafos, doctor Adrián C. Escobar, repartición de la que dependía la red radiotelefónica nacional; el Vocal del Consejo Nacional de Educación, profesor Próspero G. Alemandri; y el Inspector de Enseñanza Secundaria, dependiente del Ministerio de Justicia e Instrucción Pública, Ángel J. B. Rivera) y tendría a

1 El libro no indica fecha de edición. Nuestro ejemplar posee una dedicatoria autógrafa de Garrigós con fecha 26 de agosto de 1930. De ese mismo año son las reseñas bibliográficas que se publican en El Monitor de la Educación Común y La literatura argentina. 
su cargo "la preparación de un plan destinado a implantar progresivamente la 'Escuela del Aire', en la enseñanza primaria, secundaria, normal y especial".

Algunos meses después, en noviembre de 1939, El Monitor de la Educación Común se hace eco de la noticia y presenta en extenso las características de la Escuela: indica que la comisión ha cumplido la misión encomendada y ha presentado el plan que se resume a continuación. Según se advierte, este ha sido ya ensayado satisfactoriamente, y la Escuela comenzará a funcionar el 10 de enero de 1940. La publicación (Escuela 31-32) recuerda en primer lugar los objetivos de la flamante Escuela:

1.- Completar y perfeccionar la obra de la escuela, vinculando al alumno con el medio social en que vive.

2.- Orientar la cultura popular en todas sus manifestaciones acentuando el fondo moral y afianzando el sentimiento nacional y patriótico.

3.- Contribuir a la unidad de la enseñanza difundiendo las nociones básicas de todos los conocimientos.

Para alcanzar esos objetivos, continúa, el Plan contempla transmisiones destinadas a cinco grupos diferenciados: niños de primaria, estudiantes de enseñanza secundaria, adultos, maestros y padres. Para cada uno, propone una selección de los contenidos: mientras que las dirigidas a los estudiantes de primaria y secundaria "se referirán con preferencia a los asuntos y temas de los programas oficiales", las de adultos "comprenderán temas destinados a completar su formación cultural y su educación cívica y social”. Por su parte, aquellas dirigidas a los maestros "tenderán a perfeccionar los métodos y procedimientos de enseñanza, a informarles de las novedades en el orden científico y educacional, a dictarles instrucciones para el mejor cumplimiento y desarrollo de los programas oficiales" (32).

El Monitor expone también las características que, de acuerdo con la Comisión, debían presentar las transmisiones radiales. Destaca, en primer lugar, la exigencia de que sean "breves, variadas y amenas. Debe procurarse originalidad en la forma de presentar los asuntos" (33); un requerimiento que, como veremos, resulta determinante en la propuesta de Garrigós. En cuanto a los programas, se indica que todas las materias pueden ser motivo de transmisión, pero que "ha de darse especial preferencia a la Moral, el Lenguaje, la Historia, la Geografía, la Naturaleza y la Música" (33). Significativamente, indican también los recursos didácticos que deben utilizarse: "Lectura, Recitado, Declamación, Diálogo, Dramatización, Biografía, Conferencia, Reportaje y entrevista, Música, Canto" (33). El Monitor reproduce asimismo los programas correspondientes a los diferentes destinatarios: 18 temas para primaria, 22 para adultos, 12 para maestros y 14 para padres. En lo que respecta a los estudiantes secundarios, a quienes estaban originalmente destinadas las Lecciones de Garrigós, la Comisión no ofrece una lista de temas: señala en cambio que estas trasmisiones se realizarán dentro 
y fuera del aula, como "cursos complementarios a los establecidos en los programas oficiales" y "cursos de repaso" respectivamente (34).

\section{Las Lecciones de idioma nacional (1940)}

En 1940, apenas transcurridos unos meses de las primeras transmisiones de la Escuela, se publican las Lecciones de idioma nacional, de Florencio Garrigós. La "Introducción" del volumen está a cargo del Inspector Ángel J. B. Rivera, quien fuera parte de la Comisión a cargo de la Escuela. El texto que se presenta, recuerda Rivera, comprende las lecciones con que "fueron inauguradas el verano último las transmisiones experimentales de la Escuela del Aire, que continúan realizándose por la Radio del Estado" (5). Su publicación es consecuencia del éxito obtenido por Garrigós, quien habría conseguido un público significativamente más amplio que el previsto. Así, observa Rivera que las lecciones sobrepasaron con creces su inicial cometido pedagógico, y no solo consiguieron satisfacer las exigencias pedagógicas de la Comisión, también "las necesidades del público, no escaso, que se preocupa por mejorar el idioma” (6). Esa ampliación, para Rivera, es evidencia de la potencia expansiva de la radio y confirmación del proyecto de la Escuela: "Por eso, igeneroso destino de la Escuela del Aire!, estas lecciones no se han limitado a servir a la inteligencia de los estudiantes secundarios, sino, también, a la de todas las personas cultas, muchos profesores de la materia entre ellas, que pueden sintonizar la onda del Estado, desgraciadamente de muy corto alcance aún" (6).

Rivera señala que las lecciones de idioma nacional buscaban estimular "el repaso de los conocimientos adquiridos en el primer ańo del estudio de nuestro idioma”, y servir de introducción al estudio del segundo (6). Y subraya que la Comisión había asignado el curso a Florencio Garrigós debido a "su reconocida autoridad en la materia y su ya larga e intensa experiencia periodística y docente" (5). Así, al desplegar un discurso gramatical específico, a la vez escolar y radial, Garrigós debió contemplar los requisitos impuestos por la Comisión: "Tales requisitos no son otros que los indispensables para que la radiotelefonía cumpla su objetivo pedagógico, que consiste en auxiliar al profesor, dentro y fuera del aula" (5). Entre estos requisitos, destaca el uso del diálogo como recurso didáctico propio del formato radiofónico:

El diálogo, que se usa como medio de expresión exclusivo, es otra de las exigencias de la pedagogía radiotelefónica. [...] El profesor Garrigós interpretó magníficamente este propósito, con lo que logró que sus clases de Idioma Nacional fueran una sucesión de charlas amenísimas, en las que el oyente aprendía mucho casi sin esfuerzo. (6-7)

La cita testimonia la doble centralidad que para las autoridades educativas tenía la tarea encomendada a Garrigós. Por un lado, sus lecciones inauguran las transmisiones de la Escuela del Aire, evidencia del lugar eminente asignado a la 
cuestión del idioma. Por otro lado, la validación de unos recursos didácticos que permiten una modalidad de enseñanza ajustada a los consumos culturales de sus destinatarios, y por lo tanto un aprendizaje entretenido y "casi sin esfuerzo".

Este formato, que podemos llamar diálogo gramatical, no era completamente novedoso en la tradición gramatical escolar argentina. Lo novedoso radica aquí en el uso de la radiofonía, que imprime a esos diálogos el soporte de la lengua oral. Para dar vida a estos diálogos, las Lecciones se organizan como "una reunión de personas cultas", representadas en el texto impreso por las letras P., H., S., D. y C. ${ }^{2}$. En cada reunión, estos personajes se dedicaban a "discutir sobre distintos aspectos del lenguaje, en forma muy adecuada para atraer la atención del estudiante y del público en general", es decir, de forma "rápida, vivaz, sin ningún parlamento largo ni aburrido". Las intervenciones solo se interrumpían "para dar lugar a alguna lectura en prosa o en verso, así como a la interpretación de algún fragmento teatral, con los que se ilustraron los conceptos emitidos en el diálogo" (7).

\section{Gramática radiofónica: temas y procedimientos para un formato}

El libro se organiza en trece lecciones; cada una comprende entre tres y seis temas, que sin embargo solo se identifican en el índice y en el "Sumario" que antecede a cada lección. Cada una de estas se presenta como una conversación ininterrumpida entre los cinco personajes, que abordan los distintos contenidos sin que medie una presentación explícita; normalmente encontramos solo una referencia debida a uno de los interlocutores (frecuentemente P., el personaje a cargo de Garrigós), y el cambio de tema dentro de la misma lección se produce sin solución de continuidad. El ordenamiento de los contenidos de enseñanza obedece a un criterio aparentemente caótico: el listado completo se ofrece a continuación (Garrigós Lecciones 13):

\section{Lección primera}

a) Manejo del Diccionario. Voces anticuadas. Expresiones del arrabal.

b) Sustantivo. Aumentativos, diminutivos y despectivos.

c) El verbo. Uso de "haber" en sentido impersonal.

d) El adverbio en grado superlativo.

e) Vicios de pronunciación: supresión de las eses finales.

f) El acento en el verso.

Lección segunda

a) La prosa de Cervantes. Lectura y comentario de un trozo del Quijote.

b) Arcaísmos y abuso del gerundio.

c) Recitación de "Letanía de nuestro señor Don Quijote”, de Rubén Darío

2 Que en la versión radiofónica correspondían a los personajes interpretados por Florencio Garrigós (P), Zulema Howard (H), José Sanllorenti (S), Nora de Madrid (D) y Tulio Campos (C). 


\section{Lección tercera}

a) Valor del pretérito indefinido y del pretérito perfecto de indicativo. Uso del futuro de subjuntivo.

b) La noticia y el comentario

c) Recitación de "Al pasar", de Guido y Spano

\section{Lección cuarta}

a) Aspecto ético del lenguaje.

b) Voces eruditas y técnicas, regionales y familiares.

c) Correspondencia de los tiempos verbales en la oración compuesta.

d) La conjunción.

e) Práctica sobre la puntuación.

\section{Lección quinta}

a) Clasificación gramatical de las voces en el Diccionario.

b) Voces de acentuación dudosa.

c) La pronunciación esmerada y la pronunciación descuidada.

d) Práctica sobre la puntuación.

\section{Lección sexta}

a) Las distintas acepciones que registran las voces en el Diccionario.

b) El acento en las palabras compuestas.

c) Galicismos útiles y galicismos innecesarios.

d) Verbos transitivos que pueden emplearse como intransitivos.

e) Práctica sobre el acento.

Lección séptima

a) La lectura artística.

b) Casos especiales de concordancia del verbo con el sujeto.

c) Vicios que se cometen en la conjugación de algunos verbos de irregularidad común.

d) Italianismos naturalizados en nuestra lengua.

e) Práctica sobre el acento.

Lección octava

a) La pronunciación culta argentina.

b) Confusión de vocablos parecidos.

c) El tono del lenguaje según el asunto.

d) Empleo abusivo del participio pasivo regular.

Lección novena

a) El loísmo y el laísmo en América.

b) La prosodia y el género a través del Diccionario de la Academia. 
c) Uso incorrecto de "cuyo".

d) Verbos que solo admiten complemento directo de cosa.

e) Práctica sobre el acento.

\section{Lección décima}

a) El soneto.

b) Nombres de oficios, empleos y profesiones con terminación propia para denotar el género femenino.

c) Malos usos en la formación de algunos plurales.

d) Prosodia de los verbos terminados en "iar" y en "cuar".

e) Expresiones cuyos elementos se escriben juntos o separados, según el sentido que se les dé.

\section{Lección décimoprimera}

a) La descripción de personas, lugares y cosas.

b) Terminación genérica de los participios activos usados como nombres o adjetivos.

c) Vulgarismos producidos por síncopa, prótesis, epéntesis, aféresis, apócope y metátesis.

d) La interrogación y la admiración.

\section{Lección décimosegunda}

a) El lenguaje gauchesco. Lectura y comentario de un fragmento de Martín Fierro.

b) El yeísmo.

c) El voseo practicado por la gente culta.

Lección décimotercera

a) La narración.

b) La recitación del verso y la declamación.

c) Mal empleo del gerundio.

d) Defectos de pronunciación: falsos diptongos.

e) El paréntesis y las comillas.

El texto impreso parece replicar el guion radiofónico ${ }^{3}$; no obstante, algunas marcas, como las notas al pie que amplían los conceptos tratados o indican una fuente bibliográfica, remiten exclusivamente al universo del texto escrito. Como adelantamos, cada lección se presenta como un diálogo ininterrumpido entre

3 No nos ha sido posible hallar una grabación de las emisiones radiales. En la página web de Radio Nacional (https://www.radionacional.com.ar/audios) se conserva un fragmento de la primera lección; allí es posible verificar que el texto impreso sigue en líneas generales la emisión radiofónica, con mínimas modificaciones ("del arrabal" en el texto impreso; "arrabaleras" en la versión radiofónica). 
los distintos personajes: así, la ilación de los temas resulta de la propia dinámica de la conversación. Como se evidencia en el temario, la organización del discurso gramatical no sigue un orden como el tradicionalmente consagrado por las instituciones reguladoras (por ejemplo, por niveles gramaticales: ortografía, analogía, sintaxis) sino uno casi libre, que por una parte excede los contenidos estrictamente gramaticales; y, por otro, se desplaza creativamente entre los temas de cada lección.

Además de la modalidad del diálogo, otros procedimientos aparecen de forma recurrente. Así, y en continuidad con una tradición también ya largamente asentada en el texto gramatical escolar, en cada transmisión se leen o recitan textos literarios de diversos géneros. El texto es pródigo en la mención y ejemplificación de autores españoles, latinoamericanos y argentinos; lo distintivo no es tanto el canon que se construye, que se corresponde en lo esencial con el habitual para la época (Bombini 2004), sino el tipo de aproximación a esos textos, vinculado a las condiciones específicas del formato radiofónico.

Así, en las lecciones radiofónicas lo que se ofrece no es una experiencia de lectura sino de escucha. En esta línea, destacan los contenidos asociados directamente con la oralidad, con la recitación y la lectura en voz alta: "La lectura artística" o "El tono del lenguaje según el asunto". Las lecciones llevan a cabo toda una puesta en escena de la palabra hablada, cuya función a menudo es no solo demostrar un tipo de lectura en voz alta que se califica de adecuada sino distintas variedades de lengua, que son ejemplificadas por las intervenciones de los actores. Así, por ejemplo, se ofrece una lectura del Martín Fierro, señalando de forma explícita que es la que corresponde al género gauchesco: "Tulio Campos interpretará uno de los cantos del Martín Fierro, dándole adecuada inflexión gauchesca. De intento hemos elegido un pasaje aun no vulgarizado por los recitadores de oficio, cuya interpretación, en general, peca por lo incorrecta y exagerada" (182). A continuación, el mismo actor realiza una segunda interpretación, de otro texto literario, que se ofrece como ejemplo de una lectura correcta en la medida en que el actor consigue ofrecer una "inflexión" lingüística a la vez característica y diferenciada de la lengua gauchesca (187): "Tulio Campos interpretará una composición de José R. Luna, titulada ¿Qué más se puede pedir?, humilde y pintoresca. Noten, en el recitado, cuán distinta es la inflexión norteña de la gauchesca".

Por otro lado, hay frecuentes referencias a otros géneros menos recurridos por la tradición escolar, como el teatro argentino: un fragmento de Pa mujer, la de mi tierra, de Ernesto Marsili, permite testimoniar el habla rioplatense (124); se observa también un novedoso trabajo sobre los géneros discursivos propios de los medios de comunicación y de la prensa gráfica, como la noticia y el comentario, que formaban parte de la práctica docente de Garrigós desde hacía ya una década.

Finalmente: a pesar de su carácter de educación a distancia, las Lecciones solicitan a sus oyentes frecuentes ejercitaciones. Normalmente, hacia el final de una lección se propone un ejercicio, cuya corrección se ofrece al comenzar la siguiente. 
Los ejercicios se vinculan mayoritariamente con la ortografía, y consisten en un dictado (70): "P) Por hoy terminamos. En la próxima lección, los oyentes que lo desearen podrán hacer un ejercicio sobre puntuación. Esperamos que nos escu-

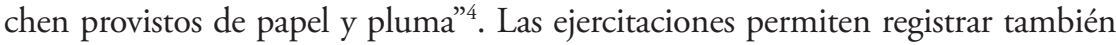
una cierta forma de interacción entre los responsables de las lecciones y sus oyentes; así, al comenzar la sexta se dice que muchos dictados han sido "devueltos por correo a sus autores" con su respectiva corrección (85). Todavía más, una lección retoma y responde una consulta de un oyente (al que, significativamente, se alude como "alumno"), referida en este caso a las reglas de acentuación gráfica (160).

\section{Corrección, norma y variedad o la audacia de las Lecciones}

El recorrido gramatical propuesto por las Lecciones incluye algunos temas que habitualmente formaban parte del curso de analogía; por ejemplo, las clases de palabras. El abordaje, sin embargo, no es exhaustivo: por una parte, no se incluyen algunas de las habituales, como el pronombre o la preposición; por otro, el tratamiento de las que se incluyen resulta al menos heterodoxo: respecto del verbo, por ejemplo, no se presenta el paradigma completo, y en cambio aparecen como temas el uso de "haber" impersonal, la distinción entre pretérito indefinido y pretérito perfecto o el uso del futuro de subjuntivo. Lo mismo ocurre con los demás niveles, la prosodia y la ortografía y la sintaxis.

En este sentido, las Lecciones no parecen obedecer a la voluntad de conseguir una explicación comprensiva. Antes bien, los contenidos privilegiados responden a una preocupación que se relaciona con el buen uso de la lengua, con la corrección lingüística. Ya desde su enunciación, los temas se ordenan en relación con un eje valorativo: "abuso del gerundio", "vicios que se cometen en la conjugación de algunos verbos de irregularidad común" o "empleo abusivo del participio pasivo regular".

Garrigós destina gran parte de las Lecciones a caracterizar con claridad el tipo de enseñanza de la norma lingüística al que aspira. En primer lugar, se trata de rechazar un tipo de policía lingüística que en las Lecciones Garrigós encarna en la figura de "Sábelotodo", pero que parece remitir también a otras relativamente contemporáneas como las de Ricardo Monner Sans o Juan B. Selva. La enseñanza de la lengua, para Garrigós, no tiene que ver con la tarea que llevan a cabo esos "celosos guardadores del idioma", cuya "manía persecutoria" rechaza de modo verdaderamente enfático (54-55):

H) ¿Han leído ustedes la severa crítica del que firma con el seudónimo de Sábelotodo?

S) No deja títere con cabeza.

H) Hablar del hablar de los demás, en un afán alocado de señalar defectos, me ha parecido tarea, si algo entretenida, de escaso provecho.

4 En las Lecciones, a diferencia evidentemente de la versión radiofónica, el texto de la ejercitación aparece ya resuelto. 
S) Sobre todo cuando adquiere los caracteres de manía persecutoria.

P) Generalmente arguye en quien la practica espíritu de intolerancia.

H) O soberbia. [...]

H) Nada ni nadie detiene a los censores del habla, tan pagados están de la función de policía que realizan.

P) Por millares podríamos contar las trasgresiones reales o imaginarias que fueron puestas en la picota, como engendros y adefesios del idioma...

H) Y que la Academia de la Lengua muy cuerdamente terminó por legitimar.

El fragmento permite ver algunas otras premisas: interesa destacar aquí ese señalamiento de que ciertos usos lingüísticos, inicialmente considerados "engendros y adefesios del idioma", fueron luego legitimados por las instituciones reguladoras, en particular por las Academias. No obstante, para Garrigós ese reconocimiento es siempre tardío, en el sentido de que los instrumentos lingüísticos como la gramática y el diccionario recogen necesariamente a posteriori aquello que antes había aparecido en el uso. Por esa razón, reivindica también (aunque indirectamente) la preparación de otro tipo de herramientas de consulta lingüística, que sirvan para "dilucidar cuantas dudas asaltan al que habla o al que escribe", una referencia obvia a su propia producción desde la década del veinte (55):

P) Muchas veces la represión [lingüística] no tiene más fundamento que el Diccionario y la gramática.

S) Muy útiles como normas de orientación.

P) Pero pobres de noticias para dilucidar cuantas dudas asaltan al que habla o al que escribe.

H) Además, no todas las voces y giros están registrados en aquellos libros.

P) Porque la Academia espera que el uso, el mejor uso los consagre como buenos para darles el pase.

La crítica de los instrumentos lingüísticos preparados por las academias, sin embargo, no solo funciona negativamente como reivindicación de esos géneros menores como los de las columnas lingüísticas en la prensa periódica; en el otro extremo, permite el ingreso al discurso gramatical escolar de las ideas introducidas en el ámbito nacional por los especialistas españoles a cargo del Instituto de Filología de la Universidad de Buenos Aires, cuyas publicaciones (de Tomás Navarro Tomás a Ramón Menéndez Pidal) se citan en varias ocasiones como fuente de autoridad.

En relación con la cuestión del correcto uso, las Lecciones establecen explícitamente el criterio de que la norma lingüística es la lengua culta, en especial aquella que se manifiesta en el uso de los mejores escritores (56): "P) En rigor, la corrección del lenguaje solo puede alcanzarse...H) Leyendo los buenos autores. P) Sí, con la observación atenta de los buenos autores". El grado de corrección de un hablante se mide, entonces, por su grado de proximidad con la variedad utilizada por esos escritores, que definirían lo que el texto denomina lenguaje culto. 
Notablemente, Garrigós cuestiona ya no a aquellos cuyo uso de la norma culta es deficiente sino a aquellos que, pudiendo usarla, deciden no hacerlo y optan en cambio por formas vulgares, "arrabaleras". La cuestión del uso así se resitúa: es ahora un problema que remite a la ética del lenguaje. O de los hablantes, como se argumenta en el siguiente diálogo (62-63):

P) Porque hay normas que ninguno tiene derecho a quebrantar.

H) Normas de buena educación.

P) Y las quebrantan quienes dicen o escriben despropósitos.

S) O emplean voces arrabaleras.

P) Hay ciertas palabras y giros confinados al habla de la gente baja, cuyo empleo no se concibe en la esfera de la gente culta.

S) En el lenguaje se refleja la buena o mala educación de las personas.

P) Por eso calificamos el lenguaje de nuestros interlocutores de culto o inculto, de urbano o incivil, según la manera como se expresan.

$\mathrm{H})$ El lenguaje tiene, pues, un fundamento ético.

S) Es decir, moral.

P) Muchas veces por una razón ética preferimos unas formas de lenguaje a otras; así haya a haiga; esté a estea.

H) Aprieta a apreta y enredo a enriedo.

S) Haya, esté, aprieta, enredo.

H) Son urbanas.

S) Las segundas: haiga, estea, apreta, enriedo.

H) Inciviles o bajas.

S) Rufino Cuervo dice con sobrada razón que el bien hablar es una de las más claras señales de la gente culta y bien nacida.

[En este y otros casos las negritas pertenecen al original]

En el fragmento anterior, los ejemplos de incorrección remiten todos al elemento verbal. Reiteradamente el texto, siguiendo como dijimos una tradición bien establecida desde el siglo XIX y antes en la gramática escolar hispánica (García Folgado 2013) se detiene en el señalamiento de esta clase de incorrecciones, frecuentemente denominadas "vicios" si remiten a la pronunciación y "vulgarismos" a la morfología verbal: "P) Trompezar y emprestar son feos vulgarismos" (15); "S) Cantastes, salistes, fuistes, hicistes. P) Es un feo vulgarismo. Lo difunden los mismos que no pronuncian las eses finales cuando deben hacerlo" (30). En varias ocasiones Garrigós acude a la germanía para caracterizar la variedad lingüística que en más alto grado encarna la incorrección lingüística, la falta de ética en el lenguaje: el arrabalero (16):

S) Aquí en vez de germanía decimos voces del arrabal o voces lunfardas.

P) Que no deben confundirse con otras de carácter familiar o festivo.

H) Así, por ejemplo, macaneo, cachada.

P) No son recomendables, pero no son del arrabal.

H) En cambio... 
S) Menega, estrilo, araca, esgunfiar, morfe, vento, rogasi...

P) Vienen del suburbio o lindan con él.

Llamativamente, las Lecciones, que se muestran deudoras de una tradición que encuentra en el arrabalero (eventualmente: en el lunfardo) su objeto de impugnación, a la vez tiene un criterio moderno en el reconocimiento de que existen otras variedades lingüísticas que exceden la distinción lenguaje culto/ lenguaje inculto. Así, en el fragmento se distingue entre formas propias de la variedad familiar ("macaneo", "cachada"), no recomendables pero tampoco incorrectas, y aquellas que, por venir del suburbio, deben ser evitadas.

Sin embargo, el aporte más significativo que las Lecciones hacen al tratamiento de la variación lingüística se relaciona con el establecimiento, contraste y legitimación de una variedad dialectal, esto es, la lengua (las lenguas, como veremos) hablada en la Argentina. Así, contra una tradición escolar que, como ha demostrado López García, buscaba frecuentemente negar o corregir los rasgos específicos de las variedades argentinas del español, el texto de Garrigós busca establecer un criterio de naturaleza opuesta.

Una y otra vez, las Lecciones se detienen en el registro de formas propias del español de la Argentina, que contrastan con las usadas en España: "P) Aquí decimos viejita y viejito, en general sin significación diminutiva. En España, los diminutivos de viejo y vieja son viejecito y viejecita, pero no viejito ni viejita" (18). El modelo de corrección, que como hemos visto Garrigós basa, por un lado, en un criterio relativamente tradicional (la lengua culta tal como se manifiesta en la literatura), se combina con un criterio geográfico (modernamente: dialectal) y de allí se sostiene que son correctos aquellos hechos de habla que pertenecen a la lengua culta hablada en el país. El criterio, como veremos, se aplica (novedosamente, para la tradición gramatical escolar argentina) a todos los niveles lingüísticos; en primer lugar, a la pronunciación (81-82):

P) Un porteño culto no dirá jamás, a no ser afectadamente: ierba mate, maio, raio, cuio.

H) Porque el yeismo porteño es un matiz de la pronunciación culta argentina.

P) Que puede convivir con la pronunciación castellana de la y griega o ye.

S) Practicada también por muchos de nuestros compatriotas.

El texto es explícito y hasta enfático en este punto: la norma lingüística no se encuentra en la pronunciación castellana, sino en la practicada por los hablantes cultos del país; todavía más, el intento de simular esa pronunciación es caracterizado como "afectado" y "falso", es decir, como violaciones a la ética del uso del lenguaje. Si en el ejemplo anterior el fenómeno que se legitimaba era el yeísmo, en el siguiente lo es el seseo (104):

S) ¿Debemos articular a la manera de los castellanos?

P) ¡Qué esperanza! En materia de pronunciación da la norma la gente culta argentina. 


\section{S) ¿Por qué razón?}

P) Por muchas. La principal consiste en que nuestro modo de hablar se diferencia del castellano; de ahí que el intento de darle un aire semejante suena siempre a afectación y falsedad.

H) Como remedo, resulta insoportable.

P) Hay otras razones, además. Algunas consonantes no se pronuncian aquí como en España.

H) Nosotros no articulamos la z.

P) Ni siquiera la s castellana, pues la emitimos con sonido distinto.

S) En conclusión, ¿cómo debemos articular?

P) Bien, es decir, sin suprimir letras y sin articularlas extrañamente, ni con esfuerzo.

Y todavía más, el texto reconoce diferencias dialectales dentro de la lengua hablada en el país, dialectos en los que incluso se prefieren formas más próximas a las de la pronunciación castellana (113):

S) Los porteńos decimos “yerro".

P) Pronunciamos la y griega como la j francesa.

S) En cambio los castellano y muchos argentinos de tierra adentro:

H) Ierro, ierras, ierra, erramos, erráis, ierran.

La cuestión se desarrolla en la octava lección: de hecho, constituye uno de sus temas, "La pronunciación culta argentina". Para Garrigós, esta pronunciación (y aquí se distancia de Menéndez Pidal, cuya interpretación cita) no es la castellana ni la andaluza culta: "P) El acento, el timbre y las inflexiones de voz nos distinguen de uno y otro y hasta presentan formas variadas en nuestro mismo territorio" (119) . La observación da paso a una intervención que reivindica la variación dialectal pero proclama a la vez la unidad lingüística y rechaza la posibilidad de un "idioma argentino" (120):

H) No faltan quienes piensan que las variantes entre el habla culta española y la nuestra conspiran contra la unidad de la lengua.

P) Es un error. Por sobre esas variantes, existe la unidad lingüística, mantenida por la acción cultural y literaria de españoles y americanos, y hasta la identidad de acento, perceptible cuando este se contrapone al acento francés, al inglés, al alemán o a otro de extraño timbre.

H) Señalar una tonalidad distinta en la pronunciación y aun en la literatura de un pueblo, no significa proclamar una escisión.

S) Ni creer por ello que se esté gestando un idioma nuevo...

5 Para Garrigós, el origen de las particularidades del habla rioplatense está en la pronunciación del gaucho: "S) De manera que los gauchos no pronunciaban la elle. P) La asimilaban a la y griega, y pronunciaban esta a la manera de los porteños, como $\mathbf{j}$ francesa. C) Práctica que hoy la siguen más de cuatro millones de argentinos. S) Yegase, mayor, ayuda, yora, hayaron, yevaba. P) En lugar de H) Llegase, maior, aiuda, llora, hallaron, llevaba" (188). Las negritas pertenecen al original. 
P) ... sino sencillamente considerar el castellano como un instrumento apto para servir las necesidades de todos los pueblos que lo hablan, sin establecer entre ellos otras preeminencias o jerarquías que las que pueden derivarse de una mayor cultura.

El reconocimiento de los fenómenos de variación, como señalamos, comprende también una serie de fenómenos léxicos y, en especial, morfosintácticos ${ }^{6}$. En esta línea, finalmente, la intervención más novedosa y arriesgada la constituye la afirmación de que el voseo es propio de la lengua culta y por lo tanto correcto (189):

P) Como todos los argentinos coetáneos y actuales, [los gauchos] usaban el vos en lugar del tú.

C) ¿Conciben Uds. que un criollo auténtico, así sea el presidente de la República, trate de tú a sus allegados?

P) La gente culta argentina, cuando habla sin afectación, usa como tratamiento ordinario el vos, porque el tú le suena a españolismo.

\section{A modo de cierre}

Como hemos sugerido, las Lecciones de Garrigós ocupan un lugar singular en la tradición argentina. Ello se debe, por una parte, a lo original del formato que les da su carácter: concebidas para ser escuchadas a distancia, constituyen una novedosa experiencia de enseñanza gramatical escolar. Por otro lado, hemos destacado también el modo en que despliegan una reflexión sobre la lengua que apunta a señalar que una serie de usos dialectales que para la tradición normativa eran sancionados como incorrectos, en la medida en que resultaban propios de la lengua culta del país, debían ser tenidos por correctos. En 1940, no se trataba de un posicionamiento sencillo: un año antes, el español Amado Alonso, director del Instituto de Filología de la Universidad de Buenos Aires desde 1927, respondía, en nombre de la Facultad de Filosofía y Letras, a una consulta realizada por la Comisión de Estudio y Reorganización de los Servicios de Radiodifusión. En un breve texto, Alonso contestaba a las cuatro preguntas formuladas por el organismo público (¿deben aceptarse en las transmisiones radiales las formas corrientes de pronunciación, como el yeísmo, o debe intentar restituirse la forma peninsular?, ¿conviene difundir comedias dialogadas en lenguaje familiar?, ¿conviene difundir teatro rural con lenguaje vernáculo?, ¿conviene difundir canciones escritas en la "jeringoza del arrabal”?); las respuestas de Alonso destacaban el carácter espiritualmente superior de la lengua general (esto es: la norma culta peninsular) y sugerían, por ejemplo, eliminar el yeísmo (8). Unos meses más tarde, las ondas radiofónicas acercaban a un amplio público una perspectiva

6 Como indicamos, no se conservan registros de las emisiones radiales, salvo un único fragmento. Allí se observa, sin embargo, que la pronunciación de los actores recurre al menos a dos fenómenos habituales del español rioplatense: el yeísmo y el seseo. 
opuesta, que además, y aquí también radica la novedad de las Lecciones, ponía en escena el espectáculo del propio uso que se buscaba legitimar.

Representante de la gramática escolar, Garrigós no se desentiende de su misión de fomentar la corrección. Y, sin embargo, de manera declarada a la vez que puesta en práctica, en las Lecciones reivindica las formas de la norma culta local como patrón de referencia para el habla sobre la que busca intervenir. Más aún, su mirada respetuosa de las variedades locales (cuyo prestigio se afirma) disipa toda crispación respecto de la amenaza de la lengua común. Así, su posición sobre la variación dialectal, que en virtud de su lugar de enunciación expresa un proyecto de Estado, se sitúa en este contraste a la vanguardia del discurso contemporáneo del campo científico, encarnado en la figura de Alonso,

En este sentido, las Lecciones son al mismo tiempo una muestra de innovación pedagógica y una señal de que el discurso gramatical escolar puede ser un vector de modernización en el saber disciplinar. Las ondas de la Escuela del Aire, así, llevan el discurso sobre el idioma nacional más lejos que lo que el campo científico habría de poder llevarlo por algunas décadas.

\section{Referencias bibliográficas}

Alonso, Amado. "El problema del idioma en la radiodifusión". Informe presentado por la Comisión de Estudio y Reorganización de los Servicios de Radiodifusión, con las opiniones recogidas en la encuesta realizada al efecto, Buenos Aires, Dirección General de Correos y Telégrafos, Talleres Gráficos de Correos y Telégrafos, 1939, pp. 8-9.

Bombini, Gustavo. Los arrabales de la literatura. La historia de la enseñanza literaria en la escuela secundaria argentina (1860-1960). Buenos Aires, Miño y Dávila, 2004.

"La escuela del aire". El monitor de la educación común, vol. LIX, n. ${ }^{\circ} 803$, nov. 1939, pp. 31-37.

García Folgado, María José. Los inicios de la gramática escolar en España (17681813). Una aproximación historiográfica. Munich, Peniope, 2013.

Garrigós, Florencio. "Encuesta de Crítica. ¿Llegaremos a tener un idioma propio? Habla el señor Florencio Garrigós (hijo)”. Crítica, 27 de junio de 1927, p. 12.

Garrigós, Florencio. Lecciones de idioma nacional. Buenos Aires, Editor Mario Tato, 1940.

Garrigós, Florencio. Gramaticales y filológicas de "La Prensa". Buenos Aires, Editor Mario Tato, 1944.

Garrigós, Florencio (h) y Francisco Camón Gálvez. Encuadernador ortográfico. Buenos Aires, Estrada, 1930. 
"Los escollos de la gramática”. El Hogar, n. ${ }^{\circ}$ 954, pp. 21 y 59.

López García, María. Nosotros, vosotros, ellos. Representaciones de la variedad rioplatense en los manuales escolares. Buenos Aires, Miño y Dávila, 2015.

Nieto, Haydée y Oscar de Majo. "La educación a distancia en Argentina". Signos Universitarios, vol. 30, n. ${ }^{\circ}$ 46, 2011, pp. 85-108.

Rivera, Ángel. "Introducción”. Lecciones de idioma nacional, Buenos Aires, Editor Mario Tato, 1940, pp. 5-8.

Reseña de El encuadernador ortográfico, de Florencio Garrigós (h) y Francisco Gamón Gálvez. El Monitor de la Educación Común, vol. 49, n. ${ }^{\circ}$ 668, 1930, pp. 294-295.

Sáenz-Hayes, Ricardo. "Palabras de homenaje a los doctores Ángel Acuña y Florencio Garrigós". Boletín de la Academia Argentina de Letras, vol. XXII, n. ${ }^{\circ}$ 84, abr.-jun. 1957, pp. 169-174. 